\title{
¿CÓMO INFLUYEN LAS PRÁCTICAS MEDIOAMBIENTALES DE RECURSOS HUMANOS EN EL RENDIMIENTO MEDIOAMBIENTAL?
}

\author{
Pablo Moura-Díez \\ Doctor en Organización de Empresas \\ Universidad Rey Juan Carlos \\ pamoudie@hotmail.com \\ Juan-José Nájera-Sánchez \\ Doctor en Ciencias Económicas y Empresariales \\ Universidad Rey Juan Carlos \\ juanjose.najera@urjc.es \\ Eva-María Mora-Valentín \\ Doctora en Dirección de Empresas \\ Universidad Rey Juan Carlos \\ evamaria.mora@urjc.es \\ Marta Ortiz-de-Urbina-Criado \\ Doctora en Dirección y Organización de Empresas \\ Universidad Rey Juan Carlos \\ marta.ortizdeurbina@urjc.es
}

\begin{abstract}
RESUMEN
La relación entre las prácticas medioambientales de recursos humanos y el rendimiento medioambiental de la empresa ha sido analizada desde un punto de vista teórico en múltiples trabajos. Sin embargo, la evidencia empírica al respecto es escasa y algunos trabajos han utilizado medidas subjetivas del rendimiento medioambiental. En este trabajo analizamos la relación entre seis prácticas medioambientales de recursos humanos (selección, formación, evaluación del rendimiento, recompensas, trabajo en equipo y participación) y el rendimiento medioambiental objetivo, medido en términos de emisiones contaminantes. Para ello, llevamos a cabo un estudio en ocho centrales de generación eléctrica, basándonos en cuestionarios, entrevistas, bases de datos de emisiones contaminantes y análisis documental. Los resultados muestran que las prácticas medioambientales de recursos humanos se encuentran asociadas con el rendimiento medioambiental. Además, encontramos que es importante tener en cuenta el nivel de actividad de la central eléctrica debido a su efecto en el nivel de emisiones.
\end{abstract}

Palabras clave: recursos humanos, gestión medioambiental, rendimiento medioambiental, sector eléctrico.

\section{HOW ENVIRONMENTAL HUMAN RESOURCE PRACTICES AFFECT THE ENVIRONMENTAL PERFORMANCE?}

\begin{abstract}
The relationship between the environmental human resources practices and the company's environmental performance has been analyzed from a theoretical point of view in multiple researches. However, the empirical evidence on the subject is scarce and some works have used subjective measures of environmental performance. In this paper, we analyze the relationship between six environmental practices of human resources (selection, training, performance evaluation, rewards, teamwork and participation) and objective environmental performance, measured in terms of pollutant emissions. To do this, we carry out a study in eight central power generation, based on questionnaires, interviews, data bases of pollutant emissions and documentary analysis. The results show that environmental human resources practices are associated with environmental performance. In addition, we found that it is important to take into account the level of activity of the power station due to its effect on the level of emissions.
\end{abstract}

Key words: human resources, environmental management, environmental performance, electricity sector. 


\section{INTRODUCCIÓN}

Una parte muy importante de la actividad económica y productiva que produce emisiones, residuos y vertidos al entorno natural se lleva a cabo en las empresas, y por ello, es necesario encontrar soluciones en el interior de las mismas (Hart, 1995; Starik y Rands, 1995). La motivación e importancia de nuestro trabajo se fundamenta en la magnitud, urgencia y relevancia de los problemas medioambientales actuales, y en el papel fundamental que juegan las organizaciones y las empresas para atenuarlo o acentuarlo. El problema es de gran calado dado que sus causas fundamentales como el ascenso de la población mundial, el aumento del consumo de recursos naturales, y el incremento del impacto medioambiental derivado de las actividades sociales y económicas, no parece que vayan a disminuir en las próximas décadas.

Las empresas pueden actuar sobre estos problemas mediante acciones tecnológicas y humanas. Ambas son muy importantes y se encuentran estrechamente relacionadas. Considerando que son las personas las que diseñan, construyen y emplean la tecnología, y toman decisiones acerca de su desarrollo, adquisición y utilización en las empresas, el interés de nuestro trabajo se centra en las prácticas medioambientales de recursos humanos. Estas prácticas persiguen mejorar los resultados de la organización en relación con el entorno natural y hacen referencia a las prácticas de selección, formación, evaluación del rendimiento, recompensas, participación y trabajo en equipo, asociadas a esta área de la empresa. Este conjunto de prácticas han sido referenciadas en la literatura como Environmental Human Resource Management Practices (Milliman y Claire, 1995; Whermeyer, 1996) o Green Human Resource Management (Jabbour y Jabbour, 2016; Mishra et al., 2014; Renwick et al., 2013).

La literatura ha abordado el estudio de estas prácticas desde una perspectiva teórica. Otros trabajos han analizado cómo se relacionan estas prácticas con la adopción de las distintas fases de un sistema de gestión medioambiental o cómo contribuyen a mejorar el rendimiento medioambiental percibido. No obstante, hay pocos trabajos que analicen su relación con el rendimiento medioambiental objetivo. Por ello, en este trabajo planteamos la siguiente pregunta de investigación: ¿Cómo se relacionan las prácticas medioambientales de recursos humanos con el rendimiento medioambiental objetivo? Para ello, englobamos las prácticas medioambientales de recursos humanos dentro del modelo capacidad, motivación y oportunidad y llevamos a cabo un estudio en ocho centrales eléctricas de ciclo combinado, con el objetivo de ver cómo se relacionan con el nivel de emisiones tóxicas de las centrales. De esta forma, se pretende contribuir a la investigación en gestión y política ambiental en las organizaciones.

\section{MARCO TEÓRICO}

En esta sección se analizan los enfoques que estudian las prácticas medioambientales de recursos humanos y el rendimiento medioambiental de la empresa.

\subsection{Prácticas medioambientales de recursos humanos}

Las prácticas se agrupan en tres grandes categorías de acuerdo al modelo capacidad, motivación y oportunidad (Appelbaum et al., 2000; Boxall y Purcell, 2003; Subramony, 2009). Dentro del mecanismo de capacidad se incluyen las actividades de reclutamiento, selección y formación medioambiental. En el mecanismo de motivación se encuentran las actividades de evaluación del rendimiento y concesión de recompensas medioambientales. Por último, en el mecanismo de oportunidad se engloban las actividades que promueven la participación y el trabajo en equipo del personal en relación a las cuestiones medioambientales. 


\subsubsection{Mecanismo de capacidad}

En la literatura sobre prácticas medioambientales de recursos humanos varios autores han propuesto que las empresas que persiguen obtener un rendimiento medioambiental superior deberían tener en cuenta las actitudes, valores, conocimientos y habilidades de los empleados en sus procesos de reclutamiento y selección (Egri y Hornal, 2002; Renwick et al., 2008; Starik y Rands, 1995). De este modo podrían incrementar las capacidades medioambientales del personal, lo que puede conducir a mejorar el rendimiento medioambiental de la empresa (Bansal, 2003; Boiral, 2002; Egri y Herman, 2000; Ramus, 2005; Rothenberg, 2003).

Esta cuestión ha sido argumentada teóricamente por varios autores que han incorporado las prácticas de reclutamiento y selección en sus modelos de prácticas medioambientales de recursos humanos. Algunos trabajos han mostrado cómo algunas empresas emplean estas prácticas (BríoGonzález et al., 2008; Jabbour et al., 2008), e incluso las han vinculado con alguna medida de rendimiento medioambiental subjetivo como la proactividad de la estrategia medioambiental o el rendimiento percibido por los directivos (Egri y Hornal, 2002; Jabbour et al., 2010). Por todo ello, es necesario analizar con más detalle la relación entre la utilización de estas prácticas y medidas de rendimiento medioambiental objetivo.

La formación es otra práctica fundamental para modelar las actitudes, proporcionar los conocimientos y construir las competencias necesarias para desarrollar una estrategia medioambiental avanzada, por lo que su papel ha sido destacado en la literatura por multitud de autores e incorporado a los principales modelos sobre prácticas medioambientales de recursos humanos (Daily y Huang, 2001; Jabbour y Santos, 2008; Milliman y Claire, 1995; Renwick et al., 2008). Muchos trabajos han considerado la formación como un factor clave para adoptar estrategias o sistemas de gestión medioambiental (Madsen y Ulhoi, 2001; Sambasivan y Fei, 2008). Otros han manifestado que la falta de formación medioambiental puede impedir a las empresas evolucionar hacia posiciones medioambientales avanzadas (Fayyazi et al., 2015; Murillo-Luna et al., 2004).

En este sentido, la literatura sobre formación medioambiental se caracteriza por ser exploratoria y descriptiva. Numerosos trabajos la analizan desde un punto de vista teórico y se apoyan en estudios de casos para analizar algunos de los aspectos clave en relación al proceso formativo (Milliman y Claire, 1995; North y Daig, 1996). Se han descrito numerosos beneficios o efectos de la formación, sus contenidos, su público objetivo y diversas cuestiones a tener en cuenta para llevar a cabo un proceso formativo eficiente (Cook y Seith, 1991; Hale, 1995; Madsen y Ulhoi, 2001; North y Daig, 1996). No obstante, la evidencia empírica respecto a la relación entre formación y rendimiento medioambiental a nivel organizativo se centra principalmente en las mejoras percibidas por directivos y empleados de empresas en las que se ha impartido la formación (Boiral, 2005; Jabbour et al., 2010; Ramus y Steger, 2000).

\subsubsection{Mecanismo de motivación}

La evaluación del rendimiento resulta necesaria para transmitir al personal que los criterios medioambientales son importantes para evaluar su desempeño en el puesto de trabajo y orientar su esfuerzo hacia los resultados medioambientales (Chinander, 2001; Jabbour y Santos, 2008). Igualmente sirve para conseguir que empleados y directivos se responsabilicen de sus objetivos y obligaciones medioambientales y garantizar así la efectividad de la gestión medioambiental (Dechant y Altman, 1994; Milliman y Clair, 1995; Opatha y Arulrajah, 2014). El sistema de evaluación es una herramienta que tiene efectos a priori, porque especifica los resultados del rendimiento esperado, y a posteriori, porque proporciona información para saber si se han cumplido ciertos objetivos y metas (Epstein y Wisner, 2005).

La literatura justifica la importancia de la evaluación del rendimiento medioambiental desde un punto de vista teórico (Chinander, 2001; Dechant y Altman, 1994; Fernández-Sánchez et al., 
2003), ofrece cierta evidencia empírica acerca de su utilización en algunas empresas (Jabbour et al., 2008; Milliman y Claire, 1995; Sharma et al., 1999) y su efecto en relación con algunos indicadores del rendimiento medioambiental (Epstein y Wisner, 2005; Jabbour et al., 2010; Sharma, 2009). No obstante, no proporciona evidencia empírica sobre la supuesta relación positiva entre la evaluación del rendimiento medioambiental del personal y el rendimiento medioambiental objetivo de la empresa.

Desde el punto de vista del medioambiente, la importancia del uso de recompensas se justifica a través de su papel para motivar al personal para llevar a cabo actividades que disminuyan el impacto medioambiental de la empresa (Daily y Huang, 2001; Jabbour y Santos, 2008; Mishra et al., 2014). A este respecto, la literatura ha ofrecido abundante argumentación teórica como la de Lothe (1999), Lothe y Myrtveit (2003) y Berrone y Gómez-Mejía (2009), fundamentada en la teoría de la agencia.

En el plano empírico, varios trabajos han aportado evidencia de la utilización de distintas recompensas, monetarias, no monetarias o ambas, en relación con las actividades medioambientales apoyándose en estudios de casos (Brío-González et al., 2008; Jabbour et al., 2008; Milliman y Clair, 1995) o en una muestra de empresas mayor (Egri y Hornal, 2002; Epstein y Wisner, 2005). Otros trabajos han considerado el efecto que tienen las recompensas sobre variables que miden el rendimiento medioambiental de forma subjetiva basándose en opiniones del personal de la empresa (Daily et al., 2007; Epstein y Wisner, 2005; Ramus y Steger, 2000). Sin embargo, pocos han evaluado el efecto que ejerce la utilización de recompensas sobre el rendimiento medioambiental objetivo (Berrone y Gómez-Mejía, 2009; Russo y Harrison, 2005).

\subsubsection{Mecanismo de oportunidad}

La literatura ha justificado desde un punto de vista teórico la importancia del trabajo en equipo para integrar y compartir información, conocimientos y experiencias procedentes de diversas áreas y niveles de la empresa $\mathrm{y}$, de este modo, poder conseguir soluciones y mejoras medioambientales (Daily y Huang, 2001; Denton, 1999; Govindarajulu y Daily, 2004). El trabajo en equipo se considera un método efectivo y apropiado para minimizar el impacto ambiental debido a que los empleados tienen un conocimiento importante sobre los procesos y productos objeto de mejora medioambiental (May y Flannery, 1995) que les permite actuar sobre las causas y posibles soluciones a determinados residuos y emisiones (Fernández-Sánchez et al., 2003; Govindarajulu y Daily, 2004). Además, son apropiados para trabajos complejos que requieren implantar programas a nivel global e implicar a miembros de varias áreas de la empresa (Denton, 1999; Hart, 1995).

En cuanto a los trabajos empíricos encontramos diversos estudios de casos que muestran la utilización del trabajo en equipo en diversas empresas, los distintos tipos y/o niveles en los que se puede utilizar y las mejoras medioambientales percibidas por los empleados y directivos (Beard y Rees, 2000; Brío-González et al., 2008; Daily et al., 2007; Hanna et al., 2000; Kitazawa y Sarkis, 2000; Remmen y Lorentzen, 2000; Strachan, 1996). A su vez, la evidencia empírica que trata de relacionar el trabajo en equipo con el rendimiento medioambiental, es escasa y se basa en medidas de rendimiento subjetivas (Jabbour et al., 2010; Sharma, 2009).

La necesidad de conseguir la participación de los empleados en los distintos programas y proyectos de mejora medioambiental se fundamenta en el hecho de que los trabajadores tienen conocimientos de distinto tipo y contenido que son necesarios para promover ideas creativas y mejorar el rendimiento medioambiental (Fernández-Sánchez et al., 2003; Kitazawa y Sarkis, 2000; Rothenberg, 2003). Además, los empleados juegan distintos papeles, asumen diferentes responsabilidades y ostentan distintas posiciones respecto a la toma de decisiones en relación a los aspectos medioambientales (Govindarajulu y Daily, 2004; Renwick et al., 2013). De esta forma, sin su apoyo e implicación no sería posible implantar las iniciativas medioambientales. La mejora continua del rendimiento medioambiental requiere un cambio cultural que fomente la participación 
de los empleados y la integración de las distintas funciones de la empresa. Si los empleados no participan, realizan sugerencias y aportan ideas, no se aprovecha plenamente su capacidad y motivación para realizar mejoras medioambientales (Fernández-Sánchez et al., 2003; Kitazawa y Sarkis, 2000).

Algunos trabajos teóricos han destacado la importancia que tiene la participación de los trabajadores para que sus conocimientos, experiencias e ideas creativas contribuyan a desarrollar en la empresa competencias ecológicas e implantar políticas y mejoras medioambientales con mayor éxito (Hart, 1995; Jackson y Seo, 2010). A su vez, los estudios de casos han demostrado cómo esta participación conduce a mejoras medioambientales (Boiral, 2005; Daily et al., 2007; Remmen y Lorentzen, 2000; Rothenberg, 2003) y algunos trabajos basados en una muestra mayor de empresas han aportado evidencia empírica acerca del efecto de la participación sobre el rendimiento medioambiental (Brío-González et al., 2007; Bunge et al., 1996; Ruiz-Quintanilla et al., 1996). No obstante, la literatura ofrece escasa evidencia empírica en torno a la relación entre la participación de los empleados y el rendimiento medioambiental objetivo.

\subsection{Rendimiento medioambiental}

En la literatura académica se han empleado medidas subjetivas y objetivas de rendimiento medioambiental. Entre las primeras, autores como Brío-González et al. (2008) se han basado en varios aspectos. Por ejemplo, si el objetivo medioambiental es cumplir o superar los mínimos establecidos por la legislación, si se pone el énfasis en las medidas correctivas o en las preventivas, si se tienen en cuenta las consideraciones medioambientales de los stakeholders, y otras acciones similares que se emplean habitualmente cuando se trata de medir la proactividad de la estrategia medioambiental (Murillo-Luna et al., 2008). Otros trabajos han considerado las intenciones de comportamiento medioambiental o la propuesta de iniciativas ecológicas por parte de los empleados (Greaves et al., 2013; Ramus y Steger, 2000), así como el grado de evolución en la adopción de un sistema de gestión medioambiental (Jabbour et al., 2008). Otros autores han analizado la percepción que tienen los directivos o los empleados sobre el rendimiento medioambiental de la empresa (Daily et al., 2007; Kaur, 2011; Paillé et al., 2014).

Entre las medidas objetivas, existen varios trabajos que tratan de medir el rendimiento medioambiental basándose en los datos de emisión de distintas substancias contaminantes proporcionados por el Toxics Release Inventory (TRI) estadounidense (Berrone y Gomez-Mejia, 2009; King y Lenox, 2000; Klassen, 2001; Klassen y Whybark, 1999; Russo, 2009; Russo y Harrison, 2005). Algunos de estos trabajos, especialmente los primeros, utilizan medidas de rendimiento medioambiental objetivo que tratan de relativizar la cantidad de emisiones tóxicas con algún indicador del tamaño empresarial o del volumen de producción. Sin embargo, esta medida no tiene en cuenta el potencial de impacto medioambiental o la peligrosidad de cada substancia (Hart y Ahuja, 1996; Klassen, 2001; Klassen y Whybark, 1999). Otros trabajos, como el de King y Lenox (2000), Russo y Harrison (2005) y Russo (2009) proponen dividir la cantidad emitida de cada substancia por el umbral de emisión a partir del cual las empresas tienen que informar al organismo correspondiente. Este umbral denominado en la literatura "cantidad reportable" (King y Lenox, 2000) es inferior para las substancias de mayor impacto y superior para las de menor impacto, por lo que se toma como un indicador que permite relativizar el impacto medioambiental en función de la substancia considerada, en lugar de asignar a todas las substancias el mismo impacto potencial (Russo, 2009). 


\section{METODOLOGÍA}

Teniendo en cuenta que hay sectores que son más sensibles a los problemas medioambientales, en este trabajo nos hemos centrado en el sector eléctrico español. En este sentido, el sector eléctrico tiene determinados efectos sobre el medio ambiente (p. ej. sobre la biodiversidad, el cambio climático, la capa de ozono, el consumo de recursos naturales, y las emisiones, residuos y vertidos) que se tratan de controlar y minimizar mediante la adopción de medidas de carácter preventivo y correctivo (Marcus y Geffen, 1998). Algunos trabajos previos también han elegido este sector para el estudio de variables medioambientales en otros países como Brasil (Pinheiro Holanda et al., 2011); mientras que otros han estudiado la importancia de las estrategias medioambientales en otros sectores como el del etanol de caña de azúcar en Brasil (Viera Peixoto Genuíno y Carvalho Machado, 2014).

Se han elegido dos de las principales empresas del sector eléctrico español, que por razones de confidencialidad de los datos, se denominarán A y B. En concreto, se ha recogido información de ocho centrales de ciclo combinado, cuatro de cada empresa participante. Las empresas A y B, incluidas en el Ibex 35, están posicionadas en los principales puestos del sector eléctrico español. La compañía A desarrolla su actividad en 22 países y está integrada por más de 17.000 personas, de las que aproximadamente el $51 \%$ desempeña su actividad en España. La compañía B cuenta con más de 25.000 empleados repartidos en 40 países. En 2010, las dos empresas tenían centrales de ciclo combinado. La tabla 1 presenta los principales datos de las centrales que han participado en este estudio.

Tabla 1. Centrales eléctricas analizadas

\begin{tabular}{|c|c|c|c|c|c|}
\hline Empresa & Central eléctrica & $\begin{array}{c}\text { Potencia } \\
\text { MW }\end{array}$ & $\begin{array}{c}\text { Nivel de } \\
\text { actividad } \\
\text { GWh }\end{array}$ & $\begin{array}{c}\text { Tamaño } \\
\mathbf{N}^{\circ} \text { grupos }\end{array}$ & Antigüedad \\
\hline \multirow{4}{*}{ A } & Central A1 & 441 & 1.401 & 1 & 1,00 \\
\hline & Central A2 & 374 & 1.838 & 1 & 4,58 \\
\hline & Central A3 & 407 & 2.183 & 1 & 8,50 \\
\hline & Central A4 & 389 & 1.497 & 1 & 2,42 \\
\hline \multirow{4}{*}{ B } & Central B1 & 400 & 1.225 & 1 & 5,42 \\
\hline & Central B2 & 831 & 1.161 & 1 & 4,08 \\
\hline & Central B3 & 424 & 1.712 & 1 & 6,50 \\
\hline & Central B4 & 378 & 704 & 1 & 8,00 \\
\hline
\end{tabular}

Fuente: elaboración propia a partir de los informes de REE (2011) y del MITYC (2011)

La recogida y análisis de la información se llevó a cabo en tres etapas. En primer lugar, se consultaron las fuentes de información secundaria disponibles. En concreto, se revisaron los informes de sostenibilidad y responsabilidad social corporativa para verificar que las empresas seleccionadas contaban con un nivel de desarrollo aceptable en materia de recursos humanos y medioambiente. Por otra parte, se recopiló evidencia relativa a las características de las centrales (tecnología, número de grupos, nivel de generación y antigüedad), disponibles en documentos e informes procedentes de Red Eléctrica de España (REE, 2011), el Ministerio de Industria, Turismo 
y Comercio (MITYC, 2011), así como de las empresas participantes. Se tomó como referencia la Ley 16/2002 para la prevención y control de la contaminación a fin de obtener los umbrales de emisiones a partir de los cuales es obligatorio reportar los niveles de emisiones de cada substancia. Finalmente, se utilizaron los archivos de datos del Registro Estatal de Emisiones y Fuentes Contaminantes para extraer los niveles de emisiones de cada una de las substancias contenidas en la legislación.

En segundo lugar, se llevó a cabo un proceso de recogida de información primaria a través de un cuestionario que se envió al departamento de recursos humanos con el objetivo de obtener información sobre la estrategia de recursos humanos y las prácticas medioambientales de recursos humanos. Las prácticas de reclutamiento y selección, formación, evaluación del rendimiento y recompensas, así como el sistema conjunto de prácticas medioambientales de recursos humanos se basan en una adaptación de la medida propuesta por Egri y Hornal (2002) para medir el grado de difusión de varias prácticas medioambientales de recursos humanos, utilizando una escala de 0 a 10 . Las prácticas de fomento de la participación y el trabajo en equipo se han construido basándose en las propuestas de Brío-González et al. (2007) y Jabbour et al. (2010), utilizando ítemes también valorados en una escala de 0 a 10 (Tabla 2 ).

Tabla 2. Prácticas de recursos humanos: variables e ítemes

\begin{tabular}{|c|c|c|c|}
\hline Mecanismos & Variables & & Ítemes \\
\hline \multirow[t]{2}{*}{ Capacidad } & $\begin{array}{l}\text { Reclutamiento y } \\
\text { selección }\end{array}$ & \multicolumn{2}{|c|}{$\begin{array}{l}\text { 1. Características personales (actitudes, valores, conocimientos y } \\
\text { habilidades) relacionados con el medioambiente en los procesos } \\
\text { de reclutamiento y selección }\end{array}$} \\
\hline & Formación & \multicolumn{2}{|c|}{ 2. La empresa proporciona formación medioambiental } \\
\hline \multirow[b]{2}{*}{ Motivación } & $\begin{array}{l}\text { Evaluación del } \\
\text { rendimiento }\end{array}$ & \multicolumn{2}{|c|}{$\begin{array}{l}\text { 3. Evaluación del rendimiento medioambiental (volumen de } \\
\text { emisiones, residuos, vertidos, consumo de materiales...) a la hora } \\
\text { de evaluar el rendimiento global }\end{array}$} \\
\hline & Recompensas & $\begin{array}{l}\text { 4. La empresa proporciona } \\
\text { recompensas monetarias } \\
\text { en función del rendimiento } \\
\text { medioambiental }\end{array}$ & $\begin{array}{l}\text { 5. La empresa proporciona } \\
\text { recompensas no monetarias } \\
\text { (reconocimientos públicos, } \\
\text { homenajes, premios) en función del } \\
\text { rendimiento medioambiental }\end{array}$ \\
\hline \multirow[b]{2}{*}{ Oportunidad } & Trabajo en equipo & \multicolumn{2}{|c|}{$\begin{array}{l}\text { 6. La empresa utiliza frecuentemente el trabajo en equipo para } \\
\text { solventar problemas medioambientales }\end{array}$} \\
\hline & Participación & \multicolumn{2}{|c|}{$\begin{array}{l}\text { 7. Los empleados participan en la resolución de problemas } \\
\text { medioambientales } \\
\text { 8. Los empleados participan en el establecimiento de objetivos } \\
\text { medioambientales }\end{array}$} \\
\hline
\end{tabular}

Fuente: elaboración propia

El rendimiento medioambiental objetivo se mide a partir del índice de emisiones tóxicas propuesto por Russo y Harrison (2005). Los índices de emisiones suelen relativizarse en base al número de trabajadores, el volumen de activos o la cifra de ventas como indicadores genéricos del tamaño o nivel de actividad de la empresa. Sin embargo, nosotros empleamos el nivel de generación en GWh, más adaptado al sector eléctrico como indicador del nivel de actividad de la central. De este modo, el índice de emisiones tóxicas relativo (IET/GWh) queda expresado de la siguiente manera:

$$
\mathrm{IET} / \mathrm{GWh}=\ln [1+\Sigma(\mathrm{Ei} / \mathrm{CRi})] / \mathrm{GWh}
$$

Donde Ei son las emisiones de la substancia i al aire, el suelo o el agua, si las emisiones están por encima de la "cantidad reportable" y cero si no lo están; CR es la "cantidad reportable", o cantidad a partir de la cual la legislación establece que una instalación debe informar sobre el nivel 
de emisión o generación de una substancia i, por haber superado su umbral mínimo a partir del cual dicha información es obligatoria; i es el indicador que denota cada una de las substancias recogidas en el Registro Estatal de Emisiones y Fuentes Contaminantes. A su vez, como se puede ver en la expresión, y en la línea de trabajos anteriores (Russo y Harrison, 2005; Russo, 2009) llevamos a cabo una transformación logarítmica, para suavizar la tendencia. Por último, medimos la antigüedad de la central por el número de años transcurridos desde su puesta en marcha.

El tercer paso fue el análisis de la información recopilada. Por una parte, se analizaron las prácticas de recursos humanos de las centrales para cada empresa así como las relaciones entre las prácticas medioambientales de recursos humanos y el rendimiento medioambiental.

\section{RESULTADOS}

Para hacer el análisis de las prácticas de recursos humanos, se ha calculado la media de las respuestas proporcionadas en cada empresa para cada práctica de recursos humanos. En la tabla 3, se resume la intensidad media con la que se emplea cada una de las prácticas medioambientales de recursos humanos. En todos los mecanismos, la empresa A presenta valores superiores, destacando el mecanismo de oportunidad con una diferencia de 3,25 puntos. Los valores más altos se presentan en ambas empresas en el mecanismo de capacidad. Para la empresa $\mathrm{B}$, las prácticas más relevantes son el reclutamiento y la selección. Mientras que la empresa A, elige un modelo en el que complementa ambas prácticas para desarrollar el mecanismo de capacidad.

En relación con el mecanismo de motivación, la empresa A da mayor importancia a la evaluación del rendimiento, siendo esta más moderada en la empresa B. Sin embargo, respecto a las recompensas, los valores que se obtienen están por debajo de 5. Para el mecanismo de oportunidad, es en la empresa A en la que más se emplea el trabajo en equipo y la participación del personal en temas medioambientales. En la empresa B, se observa una mayor preocupación por el trabajo en equipo que por la participación.

Tabla 3. Valoración de prácticas medioambientales de recursos humanos

\begin{tabular}{|c|c|c|}
\hline $\begin{array}{c}\text { Prácticas medioambientales de recursos } \\
\text { humanos }\end{array}$ & A & B \\
\hline Mecanismo de capacidad & $\mathbf{9 , 4 2}$ & $\mathbf{7 , 5 9}$ \\
\hline Reclutamiento y selección & 9,33 & 8,50 \\
\hline Formación & 9,50 & 6,67 \\
\hline Mecanismo de motivación & $\mathbf{7 , 2 5}$ & $\mathbf{4 , 7 9}$ \\
\hline Evaluación del rendimiento & 9,67 & 6 \\
\hline Recompensas & 4,83 & 3,58 \\
\hline Mecanismo de oportunidad & $\mathbf{8 , 2 5}$ & $\mathbf{5 , 0 0}$ \\
\hline Trabajo en equipo & 8 & 6 \\
\hline Participación & 8,50 & 4 \\
\hline
\end{tabular}

Fuente: elaboración propia

Para medir la relación entre las prácticas medioambientales de recursos humanos y el rendimiento medioambiental se ha utilizado el coeficiente de correlación de Pearson. El coeficiente de correlación entre las prácticas medioambientales de recursos humanos y el índice de emisiones tóxicas relativo se sitúa en $-0,8438$, estadísticamente significativo al $99 \%$ de confianza. Si se analiza la relación para cada mecanismo y para cada práctica, todas las correlaciones son estadísticamente significativas (99\%), siendo la más alta la del mecanismo de oportunidad $(-0,8421)$ y dentro de éste, el trabajo en equipo con una correlación de $-0,8765$; mientras que la participación tiene una 
correlación menor $(-0,8230)$. Para el mecanismo de capacidad, la correlación es de $-0,8377$; y dentro de éste, la formación presenta la correlación más alta $(-0,8396)$, siendo la de reclutamiento y selección de -0,8311. Finalmente, la correlación del mecanismo de motivación es de -0,8338. Tanto la evaluación del rendimiento como las recompensas tienen una correlación de $-0,8452$. Esto indica que hay una relación inversa entre ellas, es decir, cuanto mayor es la intensidad de las prácticas medioambientales de recursos humanos, menor es el índice de emisiones tóxicas.

A su vez, el coeficiente de correlación entre el nivel de generación y el índice de emisiones tóxicas relativo se sitúa en $-0,7220$, significativo al $95 \%$ de confianza. En este primer análisis, el nivel de generación de las cuatro centrales de la empresa A es de 6.919 GWh y el de las cuatro centrales de la empresa B es de $4.802 \mathrm{GWh}$, lo que supone una diferencia de $2.117 \mathrm{GWh}$. Considerando que el nivel de generación muestra una correlación estadísticamente significativa con el rendimiento medioambiental, realizamos un segundo análisis eliminando las centrales que introducen mayor dispersión en cuanto al nivel de generación. Para ello, eliminamos la central de la empresa A que presenta mayor actividad y la central de la empresa B con menor actividad. De este modo, las tres centrales de A cuentan con un nivel de generación de $4.736 \mathrm{GWh}$ y las tres centrales de B muestran una actividad de $4.098 \mathrm{GWh}$, por lo que la diferencia se reduce desde los $2.117 \mathrm{GWh}$ hasta los $638 \mathrm{GWh}$.

En este segundo análisis, integrado por seis centrales más similares, el coeficiente de correlación entre las prácticas medioambientales de recursos humanos y el índice de emisiones tóxicas relativo alcanza un valor de $-0,8140$, estadísticamente significativo al $95 \%$ de confianza; mientras que el nivel de generación muestra un coeficiente de $-0,4782$ con el índice de emisiones tóxicas relativo, que no es estadísticamente significativo. En este caso, se observa que para las diferentes prácticas de recursos humanos, las correlaciones con el índice de emisiones tóxicas relativo también son altas y estadísticamente significativas al 95\%. La correlación más alta se da en el mecanismo de oportunidad $(-0,8114)$, seguido por el mecanismo de capacidad $(-0,8079)$ y el de motivación $(-0,8032)$. La práctica de recursos humanos con la correlación más elevada es el trabajo en equipo $(-0,8320)$, siendo la siguiente las recompensas $(-0,8178)$. Por su parte, la correlación para la formación es de $-0,8089$ y para reclutamiento y selección de -0,8040. Las correlaciones más bajas se observan para la participación $(-0,8005)$ y para la evaluación del rendimiento $(-0,7981)$.

\section{DISCUSIÓN Y CONCLUSIONES}

Este trabajo realiza una aportación a la literatura al analizar el efecto individual y conjunto de todas las prácticas medioambientales de recursos humanos sobre el rendimiento medioambiental, utilizando medidas objetivas. Se observa que las empresas que desarrollan más este tipo de prácticas presentan mejores resultados medioambientales.

La literatura sobre recursos humanos ha puesto de manifiesto que las prácticas de recursos humanos en su conjunto se refuerzan mutuamente y crean un efecto sinérgico que permite obtener mejores resultados (Paauwe, 2009; Subramony, 2009). Los trabajos que han evaluado las prácticas medioambientales desde una perspectiva de conjunto han utilizado medidas subjetivas como el rendimiento medioambiental percibido (Daily y Huang, 2007; Kaur, 2011; Paillé et al., 2014), los beneficios organizativos percibidos (Egri y Hornal, 2002), las iniciativas ecológicas propuestas por los empleados (Ramus y Steger, 2000), la consecución de una ventaja competitiva basada en la acción medioambiental (Brío-González et al., 2007) o los cambios en la adopción del sistema de gestión medioambiental (Jabbour et al., 2008, 2010), todas ellas basadas en la opinión de directivos o empleados.

Por otra parte, la literatura previa ha analizado el efecto individual de alguna práctica medioambiental de recursos humanos sobre el rendimiento medioambiental empleando medidas de carácter objetivo. Así por ejemplo, los trabajos de Berrone y Gómez-Mejía (2009) y Russo y Harrison (2005) vinculan las recompensas con el rendimiento medioambiental. Bunge et al. (1996) 
y Ruiz-Quintanilla et al. (1996) analizan la relación entre la participación de los empleados y el rendimiento.

Los resultados obtenidos para el mecanismo de capacidad ofrecen nueva evidencia empírica que avala la consideración que hacen otros autores sobre el hecho de que las empresas que persiguen obtener un rendimiento medioambiental superior deberían tener en cuenta las actitudes, valores, conocimientos y habilidades de los empleados en sus procesos de reclutamiento y selección (Egri y Hornal, 2002; Renwick et al. 2008; Starik y Rands, 1995). Para el caso de la formación, los resultados aportan nueva evidencia que es coherente con los estudios previos que han encontrado una relación entre la formación y rendimiento medioambiental percibido (Boiral, 2005; Jabbour et al., 2010; Ramus y Steger, 2000).

Para el mecanismo de motivación, los resultados obtenidos para la evaluación del rendimiento complementan los resultados obtenidos en la literatura previa (p. ej. Epstein y Wisner, 2005; Jabbour et al., 2010; Sharma, 2009) y apoyan la idea de que la evaluación del rendimiento es una herramienta que puede ayudar a orientar el esfuerzo de los trabajadores hacia la mejora de los resultados medioambientales (Chinander, 2001; Jabbour y Santos, 2008). Para el caso de las recompensas, los resultados ofrecen nueva evidencia empírica que complementa a la ya existente (p. ej. Berrone y Gómez-Mejía, 2009; Russo y Harrison, 2005). En este sentido, se refuerza la idea de que las recompensas pueden motivar la realización de actividades que disminuyan el impacto medioambiental de la empresa (Daily y Huang, 2001; Jabbour y Santos, 2008; Mishra et al., 2014).

Finalmente, para el mecanismo de oportunidad, el estudio del efecto del trabajo en equipo ofrece resultados que complementan los obtenidos en trabajos previos en los que se emplean medidas del rendimiento subjetivas (Jabbour et al., 2010; Sharma, 2009). Esto refuerza la idea de que el trabajo en equipo puede ayudar a encontrar soluciones para los problemas medioambientales (Daily y Huang, 2001; Denton, 1999; Govindarajulu y Daily, 2004). Para el caso de la participación, los resultados también ofrecen nueva evidencia empírica que complementa a la ya existente (Brío-González et al., 2007; Bunge et al., 1996; Ruiz-Quintanilla et al., 1996). En este sentido, la participación de los empleados en los distintos programas y proyectos de mejora medioambiental puede promover el desarrollo de ideas creativas y ayudar a mejorar el rendimiento medioambiental (Fernández-Sánchez et al., 2003; Kitazawa y Sarkis, 2000; Rothenberg, 2003).

En este trabajo se ha mostrado el interés que tiene el estudio del efecto de las prácticas medioambientales de recursos humanos, agrupadas sobre la base del modelo capacidad, motivación y oportunidad, sobre el rendimiento medioambiental objetivo. Teniendo en cuenta la literatura previa y la naturaleza exploratoria de este trabajo, se propone el siguiente modelo de análisis (Figura 1). El modelo plantea la necesidad de analizar de forma individual el efecto causal (positivo, negativo o neutro) que tiene cada práctica medioambiental en el rendimiento medioambiental. Esta información, en un mundo caracterizado por la escasez de recursos, debería permitir una mejor orientación de los esfuerzos hacia aquellas prácticas de recursos humanos más beneficiosas. También, puede ser interesante observar el efecto conjunto de todas o algunas de las prácticas sobre el rendimiento medioambiental. Por ejemplo, ¿tiene la inversión en formación un efecto directo sobre la mejora del rendimiento medioambiental si no se realizan buenas prácticas de evaluación del rendimiento y compensación? 
Figura 1: Modelo de análisis

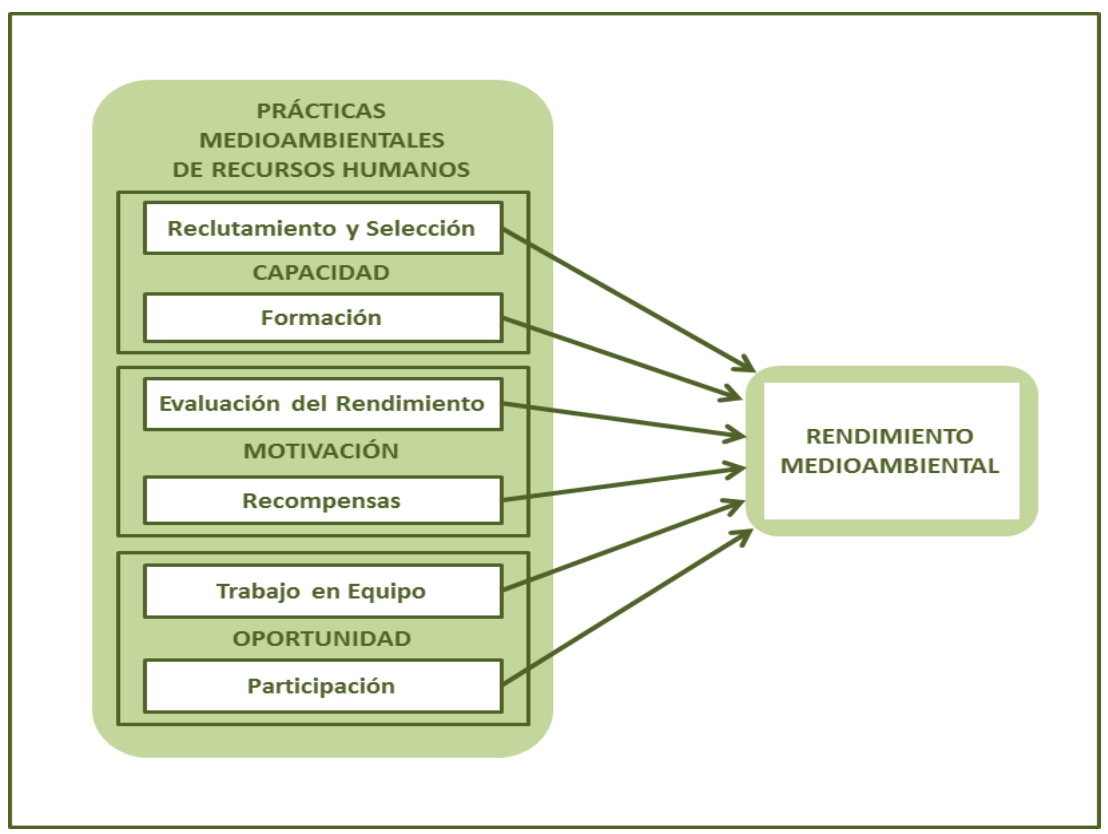

Fuente: elaboración propia

Este modelo sugiere que los directivos que quieran aprovechar el potencial de sus plantas productivas en cuanto al rendimiento medioambiental deberían invertir no sólo en medidas tecnológicas sino también en humanas. Concretamente, el presente trabajo muestra que cuando se llevan a cabo actividades como el reclutamiento y la selección, la formación, la evaluación del rendimiento, o las recompensas en materia medioambiental se puede influir en el rendimiento medioambiental de la empresa. Esto puede ser especialmente interesante para aquellos directivos con responsabilidades medioambientales y de recursos humanos que operen en sectores sensibles a los temas medioambientales y que reciban presiones significativas de los stakeholders para mejorar el rendimiento medioambiental.

Por otra parte, futuros trabajos deberían realizar investigaciones que permitan establecer relaciones causa-efecto entre las prácticas medioambientes de recursos humanos y el rendimiento medioambiental objetivo, utilizando muestras grandes y en la medida de lo posible, análisis longitudinales.

Finalmente, otros trabajos podrían analizar el modelo propuesto en otros países y sectores de la economía. En este sentido, la actividad económica en los países desarrollados está estrechamente relacionada con la pobreza y la degradación de los menos desarrollados. Una estrategia de desarrollo sostenible debe de reconocer esta relación, reduciendo el impacto medioambiental y aumentando los beneficios económicos de los mercados menos desarrollados que se ven afectados por las actividades empresariales (Hart y Dowell, 2011). Todo ello implica trabajar durante un periodo prolongado para desarrollar y desplegar tecnologías y competencias de bajo impacto ambiental, especialmente, en los mercados emergentes.

\section{AGRADECIMIENTOS}

Este trabajo ha sido apoyado por el proyecto ECO2015-67434-R del Ministerio de Economía y Competitividad (España) y por el Grupo de Investigación de Excelencia "Strategor" URJC-Banco de Santander. 


\section{BIBLIOGRAFÍA}

Appelbaum, E., Bailey, T., Berg, P., \& Kalleberg, A. L. (2001). Do high performance work systems pay off? Research in Sociology of Work, 10, 85-108.

Bansal, P. (2003). From issues to actions: The importance of individual concerns and organizational values in responding to natural environmental issues. Organization Science, 14(5), 510-527.

Beard, C., \& Rees, S. (2000). Green teams and the management of environmental change in a UK county council. Environmental Management and Health, 11(1), 27-38.

Berrone, P., \& Gomez-Mejia, L. R. (2009). Environmental performance and executive compensation: An integrated agency-institutional perspective. Academy of Management Journal, 52(1), 103-126.

Boiral, O. (2002). Tacit knowledge and environmental management. Long Range Planning, 35(3), 291-317.

Boiral, O. (2005). The impact of operator involvement in pollution reduction: Case studies in canadian chemical companies. Business Strategy and the Environment, 14(6), 339-360.

Boxall, P., \& Purcell, J. (2003). Strategy and Human Resource Management. Basingstoke and New York: Palgrave Macmillan.

Brío-González, J. A., Fernández-Sánchez, E., \& Junquera-Cimadevilla, B. (2007). Management and employee involvement in achieving an environmental action-based competitive advantage: An empirical study. International Journal of Human Resource Management, 18(4), 491-522.

Brío-González, J. A., Junquera-Cimadevilla, B., \& Ordiz-Fuertes, M. (2008). Human resources in advanced environmental approaches-a case analysis. International Journal of Production Research, 46(21), 6029-6053.

Bunge, J., Cohen-Rosenthal, E., \& Ruiz-Quintanilla, A. (1996). Employee participation in pollution reduction: Preliminary analysis of the Toxics Release Inventory. Journal of Cleaner Production, 4(1), 9-16.

Chinander, K. R. (2001). Aligning accountability and awareness for environmental performance in operations. Production and Operations Management, 10(3), 276-291.

Cook, J., \& Seith, B. J. (1991). Environmental training: A tool for assuring compliance. Journal of Environmental Regulation, 1(2), 167-172.

Daily, B. F., Bishop, J. W., \& Steiner, R. (2007). The mediating role of EMS teamwork as it pertains to HR factors and perceived environmental performance. Journal of Applied Business Research, 23(1), 95-109.

Daily, B. F., \& Huang, S. (2001). Achieving sustainability through attention to human resource factors in environmental management. International Journal of Operations \& Production Management, 21(12), 1539-1552. 
Dechant, K., \& Altman, B. (1994). Environmental leadership: From compliance to competitive advantage. Academy of Management Executive, 8(3), 7-27.

Denton, D. K. (1999). Employee involvement, pollution control and pieces to the puzzle. Environmental Management and Health, 10(2), 105-111.

Egri, C. P., \& Herman, S. (2000). Leadership in the North American environmental sector: Values, leadership styles, and contexts of environmental leaders and their organizations. Academy of Management Journal, 43(4), 571-604.

Egri, C., \& Hornal, R. (2002). Strategic environmental human resource management and perceived organizational performance: An exploratory study of the Canadian manufacturing sector. In Sanjay Sharma Y Mark Starik (Eds.), Research in corporate sustainability: The evolving theory and practice of organizations in the natural environment (pp. 205-236). Cheltenham, UK: Edward Elgar.

Epstein, M. J., \& Wisner, P. S. (2005). Managing and controlling environmental performance: Evidence from Mexico. Advances in Management Accounting, 14, 115-137.

Fayyazi, M., Shahbazmoradi, S., Afshar, Z., \& Shahbazmoradi, M. (2015). Investigating the barriers of the green human resource management implementation in oil industry. Management Science Letters, 5(1), 101-108.

Fernández-Sánchez, E., Junquera-Cimadevilla, B., \& Ordiz-Fuertes, M. (2003). Organizational culture and human resources in the environmental issue: A review of the literature. International Journal of Human Resource Management, 14(4), 634-656.

Govindarajulu, N., \& Daily, B. F. (2004). Motivating employees for environmental improvement. Industrial Management and Data Systems, 104, 364-372.

Greaves, M., Zibarras, L. D., \& Stride, C. (2013). Using the theory of planned behavior to explore environmental behavioral intentions in the workplace. Journal of Environmental Psychology, 34, 109-120.

Hale, M. (1995). Training for environmental technologies and environmental management. Journal of Cleaner Production, 3(1-2), 19-23.

Hanna, M. D., Newman, W. R., \& Johnson, P. (2000). Linking operational and environmental improvement through employee involvement. International Journal of Operations and Production Management, 20(2), 148-165.

Hart, S. L. (1995). A natural resource based view of the firm. Academy of Management Review, 20(4), 986-1014.

Hart, S. L., \& Ahuja, G. (1996). Does it pay to be green? An empirical examination of the relationship between emission reduction and firm performance. Business Strategy and the Environment, 5(1), 30-37.

Hart, S. L., \& Dowell, G. (2011). A natural-resource-based view of the firm: Fifteen years after. Journal of Management, 37(5), 1464-1479. 
Jabbour, C. J. C., \& Jabbour, A. B. L. (2016). Green human resource management and green supply chain management: Linking two emerging agendas. Journal of Cleaner Production, 112, 18241833.

Jabbour, C. J. C., \& Santos, F. C. A. (2008). Relationships between human resource dimensions and environmental management in companies: Proposal of a model. Journal of Cleaner Production, 16(1), 51-58.

Jabbour, C. J. C., Santos, F. C. A., \& Nagano, M. S. (2008). Environmental management system and human resource practices: Is there a link between them in four Brazilian companies? Journal of Cleaner Production, 16(17), 1922-1925.

Jabbour, C. J. C., Santos, F. C. A., \& Nagano, M. S. (2010). Contributions of HRM throughout the stages of environmental management: Methodological triangulation applied to companies in Brazil. International Journal of Human Resource Management, 21(7), 1049-1089.

Jackson, S. E., \& Seo, J. (2010). The greening of strategic HRM scholarship. Organization Management Journal, 7(4), 278-290.

Kaur, H. (2011). Impact of human resource factors on perceived environmental performance: An empirical analysis of a sample of ISO 14001 EMS companies in Malaysia. Journal of Sustainable Development, 4(1), 211-224.

King, A. A., \& Lenox, M. J. (2000). Industry self-regulation without sanctions: The chemical industry's responsible care program. Academy of Management Journal, 43(4), 698-716.

Kitazawa, S., \& Sarkis, J. (2000). The relationship between ISO 14001 and continuous source reduction programs. International Journal of Operations and Production Management, 20(2), 225248.

Klassen, R. D. (2001). Plant-level environmental management orientation: The influence of management views and plant characteristics. Production and Operations Management, 10(3), 257275.

Klassen, R. D., \& Whybark, D. C. (1999). The impact of environmental technologies on manufacturing performance. Academy of Management Journal, 42(6), 599-615.

Lothe, S. (1999). Compensation systems for improving environmental performance. Business Strategy and the Environment, 8(6), 313-321.

Lothe, S., \& Myrtveit, I. (2003). Compensation systems for green strategy implementation: Parametric and non-parametric approaches. Business Strategy and the Environment, 12(3), 191-203.

Madsen, H., \& Ulhoi, J. P. (2001). Greening of human resources: Environmental awareness and training interests within the workforce. Industrial Management and Data Systems, 100(1), 57-63.

Marcus, A., \& Geffen, D. (1998). The dialectics of competency acquisition: Pollution, prevention in electric generation. Strategic Management Journal, 19(12), 1145-1168.

May, D. R., \& Flannery, B. L. (1995). Cutting waste with employee involvement teams. Business Horizons, 38(5), 28-38. 
Milliman, J., \& Clair, J. (1995). Environmental HRM practices in the USA: A review of the literature. Greener Management International, 10, 34-48.

Mishra, R., Sarkar, S., \& Kiranmai, J. (2014). Green HRM: Innovative approach in Indian public enterprises. World Review of Science, Technology and Sustainable Development, 11(1), 26-42.

MITYC (2011). La Energía en España 2010. Ministerio de Industria, Turismo y Comercio. Secretaría de Estado de Energía.

Murillo-Luna, J. L., Garcés-Ayerbe, C., \& Rivera-Torres, P. (2004). Estrategia empresarial y medio ambiente: Opinión de un grupo de expertos. Universia Business Review, 4, 52-63.

Murillo-Luna, J. L., Garcés-Ayerbe, C., \& Rivera-Torres, P. (2008). Why do patterns of environmental response differ? A stakeholders' pressure approach. Strategic Management Journal, 29(11), 1225-1240.

North, K., \& Daig, S. (1996). Environmental training in British and German companies. In Walter Wehrmeyer (Ed.), Greening People: Human Resources and Environmental Management. Sheffield, England: Greenleaf Publishing.

Opatha, H., \& Arulrajah, A. A. (2014). Green human resource management: Simplified general reflections. International Business Research, 7(8), 101-112.

Paauwe, J. (2009). HRM and performance: Achievements, methodological issues and prospects. Journal of Management Studies, 46(1), 129-142.

Paillé, P., Chen, Y., Boiral, O., \& Jin, J. (2014). The impact of human resource management on environmental performance: An employee-level study. Journal of Business Ethics, 121(3), 451-466.

Pinheiro Holanda, A., Ruiz de Almada, S., Martins Mendes De Luca, \& Vasconcelos Gallon, A. (2011). O Desempenho Socioambiental nas empresas do sector eléctrico brasileiro: Uma questão relevante para o desempenho financiaro? Revista de Gestão Social e Ambiental, 5(3), 53-72.

Ramus, C. A. (2005). Context and values: Defining a research agenda for studying employee environmental motivation in business organizations. In Sanjay Sharma \& J. A. Aragón-Correa (Eds.), Corporate Environmental Strategy and Competitive Advantage (pp. 71- 95). Cheltenham, UK: Edward Elgar.

Ramus, C. A., \& Steger, U. (2000). The roles of supervisory support behaviors and environmental policy in employee" ecoinitiatives" at leading-edge European companies. Academy of Management Journal, 43(4), 605-626.

REE (2011). El Sistema Eléctrico Español 2010. Red Eléctrica de España.

Remmen, A., \& Lorentzen, B. (2000). Employee participation and cleaner technology: Learning processes in environmental teams. Journal of Cleaner Production, 8(5), 365-373.

Renwick, D. W. S., Redman, T., \& Maguire, S. (2008). Green HRM: A review, process model, and research agenda. Discussion Paper No 2008.01 April 2008. 
Renwick, D. W., Redman, T., \& Maguire, S. (2013). Green human resource management: A review and research agenda. International Journal of Management Reviews, 15(1), 1-14.

Rothenberg, S. (2003). Knowledge content and worker participation in environmental management at NUMMI. Journal of Management Studies, 40(7), 1783-1802.

Ruiz-Quintanilla, S. A., Bunge, J., Freeman-Gallant, A., \& Cohen-Rosenthal, E. (1996). Employee participation in pollution reduction: A socio-technical perspective. Business Strategy and the Environment, 5(3), 137-144.

Russo, M. V. (2009). Explaining the impact of ISO 14001 on emission performance: A dynamic capabilities perspective on process and learning. Business Strategy and the Environment, 18, 307319.

Russo, M. V., \& Harrison, N. S. (2005). Organizational design and environmental performance: Clues from the electronics industry. Academy of Management Journal, 48(4), 582-593.

Sambasivan, M., \& Fei, N. Y. (2008). Evaluation of critical success factors of implementation of ISO 14001 using analytic hierarchy process (AHP): A case study from Malaysia. Journal of Cleaner Production, 16(13), 1424-1433.

Sharma, S., Pablo, A. L., \& Vredenburg, H. (1999). Corporate environmental responsiveness strategies the importance of issue interpretation and organizational context. Journal of Applied Behavioral Science, 35(1), 87-108.

Sharma, S. (2009). The mediating effect of information availability between organization design variables and environmental practices in the Canadian hotel industry. Business Strategy and the Environment, 18(4), 266-276.

Starik, M., \& Rands, G. P. (1995). Weaving an integrated web: Multilevel and multisystem perspectives of ecologically sustainable organizations. Academy of Management Review, 20(4), 908-935.

Strachan, P. A. (1996). Achieving environmental excellence through effective teamwork. Team Performance Management, 2(1), 25-29.

Subramony, M. (2009). A meta-analytic investigation of the relationship between HRM bundles and firm performance. Human Resource Management, 48(5), 745-768.

Vieira Peixoto Genuíno, S. L., \& Carvalho Machado, A. G. (2014). Motivações e modos de formação de estratégias ambientais no setor sucroalcooleiro Brasileiro. Revista de Gestão Social e Ambiental, 8(2), 76-93.

Wehrmeyer, W. (1996). Greening People: Human Resources and Environmental Management. Sheffield, England: Greenleaf Publis

Data da submissão: 28/04/2016

Data da publicação: 20/12/2016 